\title{
Planning Aspects of Volumetric Modulated Arc Therapy and Intensity Modulated Radio therapy in Carcinoma Left Breast - A Comparative Study
}

\author{
Varadharajan Ekambaram ${ }^{1,2} *$, Ramasubramanian Velayudham ${ }^{2}$, Shiyama \\ Swaminathan ${ }^{1}$, Padmanabhan Loganathan ${ }^{1}$,Vijaya Swaminathan ${ }^{1}$
}

\begin{abstract}
Background: The advantages of Rapid Arc plans versus Intensity modulated radiotherapy plans for Carcinoma left breast were analyzed. Materials and Methods: In this study 20 Post mastectomy carcinoma left breast patients were analyzed. Both Intensity modulated Radiotherapy and Rapid Arc plans were generated for these patients. IMRT plans with 7 beams in an arc fashion and VMAT plans with two semi arcs were made to achieve $95 \%$ dose coverage to $100 \%$ volume. The plans were evaluated using Dose volume Histograms. Results: The mean Conformity and Homogeneity index in VMAT is found to be 1.05 and 0.065 respectively whereas in IMRT it was 1.07 and 0.069 . The $20 \%$ volume of Heart received a mean dose of 960cGy in VMAT and 1300cGy in IMRT. The mean dose was 1236cGy in VMAT and 1870cGy in IMRT. The ipsilateral Lung received 3395cGy to $5 \%$ volume and $1840 \mathrm{cGy}$ to $20 \%$ volume on an average and the mean dose was $1205 \mathrm{cGy}$ in VMAT, while the same were found to be $3525 \mathrm{cGy}, 2012 \mathrm{cGy}$ and $1435 \mathrm{cGy}$ respectively in IMRT. The Contralateral Lung received a mean dose of 505cGy in VMAT and 553cGy in IMRT. The mean Monitor units in VMAT were 512MU and 1170MU in IMRT. The NTID in VMAT is $108.8 \times 10^{5} \mathrm{Gycm}^{3}$ and $110.1 \times 10^{5} \mathrm{Gycm}^{3}$ in IMRT. Conclusions: The target coverage, homogeneity and Conformity index were better in VMAT plans. The Ipsilateral Lung and heart dose were very less in VMAT plans. The Contralateral Lung dose and the Normal Tissue Integral Dose were also lesser in VMAT plans however the difference is not very appreciable. The MU in VMAT plans is almost $50 \%$ that of the IMRT plans which results in the reduction of treatment time. On the whole VMAT proves to be a better modality for treating Ca. Left Breast Patients.
\end{abstract}

Keywords: Volume modulated arc therapy - breast cancer - intensity modulated radiotherapy,

Asian Pac J Cancer Prev, 16 (4), 1633-1636

\section{Introduction}

Breast cancer is the most common malignancy in women. Radiotherapy is an integral part of Breast cancer management either after breast conservation surgery or in post mastectomy cases. Many prospective studies have shown that adjuvant radiotherapy improves local control and survival rate in breast cancer patients after surgery.

There have been significant advances in the delivery of radiotherapy over the past few decades. These include increased sophistication of imaging techniques, which has resulted in improved accuracy of target volume definition and delineation, as well as developments in treatment planning systems and linear accelerator delivery capabilities thus leading to improved dose distributions and conformity. These developments have been mainly driven by the need to reduce the dose to normal tissue structures and thereby minimizing the risk of toxicity and morbidity, which then allows dose escalation to the tumor volume potentially leading to improved loco regional control. To that end, newer radiation techniques, e.g. intensity modulated radiotherapy (IMRT), has been developed (Moorthy et al., 2013; Safae et al., 2014)

IMRT techniques employ variable intensity across multiple radiation beams leading to the construction of highly conformal dose distributions. This is achieved by subdividing each radiation beam into smaller radiation beamlets and varying the individual intensities of these beamlets. The advantages of this technique is improved target volume conformity, particularly in volumes with complex concave shapes, and improved sparing of normal tissues and organs at risk (OARs) resulting in reduced acute and late toxicities. IMRT also has the ability to produce inhomogeneous dose distributions, which allows

${ }^{1}$ Dept. of Radiation Oncology, Billroth Hospitals Ltd, Shenoy Nagar, Chennai, ${ }^{2}$ Nuclear and Medical Physics Division, School of Advanced Sciences, VIT University, Vellore,Tamil Nadu, India*For correspondence: evaradharajan@gmail.com,varadharajan78@ rediffmail.com 
Varadharajan Ekambaram MSc et al

the simultaneous delivery of different doses per fraction to separate areas within the target volume(Wei-Guo Zhu et al., 2012).

VMAT was first introduced in 2007 and described as a novel radiation technique that allows simultaneous variation of three parameters during treatment delivery, i.e. gantry rotation speed, treatment aperture shape via movement of MLC leaves and dose rate. Volumetric modulated arc therapy (VMAT) is a novel radiation technique, which can achieve highly conformal dose distributions with improved target volume coverage and sparing of normal tissues compared with conventional radiotherapy techniques. VMAT also has the potential to offer additional advantages, such as reduced treatment delivery time compared with conventional static field intensity modulated radiotherapy (IMRT)(Nicolini et al., 2009; Naqvi et al., 2010; Rana, 2013). Many studies pertaining to VMAT are being carried out in different sites to study its advantage (Swamy et al., 2014)

Before implementing any new modality it is essential to evaluate its efficacy and limitations in executing it in different clinical situations. Both physical and radiobiological evaluation plays a vital role. Before implementing IMRT, its efficacy in treating different cases in planning as well as the radiobiological advantage was analyzed in our institute (Ekambaram et al., 2014; Ekambaram and Velayudham, 2014). Now when we installed VMAT in our institute, we have proceeded with its commissioning as well as evaluated its performance in different clinical situations (Ekambaram and Velayudham, 2013).

Purpose of the study: In our Centre we have recently upgraded our existing linear accelerator with IMRT facility to Rapid Arc modality. Since this is a newer facility in our institute we made many feasibility studies to analyze its efficacy in different clinical situations. In this study we have made an attempt to compare the advantages of Rapid Arc plans versus Intensity modulated radiotherapy plans for Carcinoma left breast.

\section{Materials and Methods}

In this treatment planning study we have analyzed about 20 Post mastectomy left breast carcinoma patients. All the patients were immobilized using semi body Vaclok Immobilization system. A $0.5 \mathrm{~cm}$ Bolus is placed on the skin in the treatment area. Patients were positioned on a wide bore Siemens CT Simulator flat couch with the help of lasers and both arms of the patient were raised above patient's head. Furthermore, radio opaque markers were placed during the immobilization procedure to guide the isocenter shift. For all the patients, CT scan images with as well as without bolus were obtained from mandible to upper abdomen area with intravenous contrast, and CT scans were obtained using slice thickness of $3 \mathrm{~mm}$. After CT simulation, DICOM images were transferred to Eclipse Treatment Planning System (version 10.0 Varian Medical Systems, Palo Alto, California, USA). Planning Target Volumes and Organs at Risk were delineated on the axial CT slices.

For treatment planning, $6 \mathrm{MV}$ X-rays from Clinac

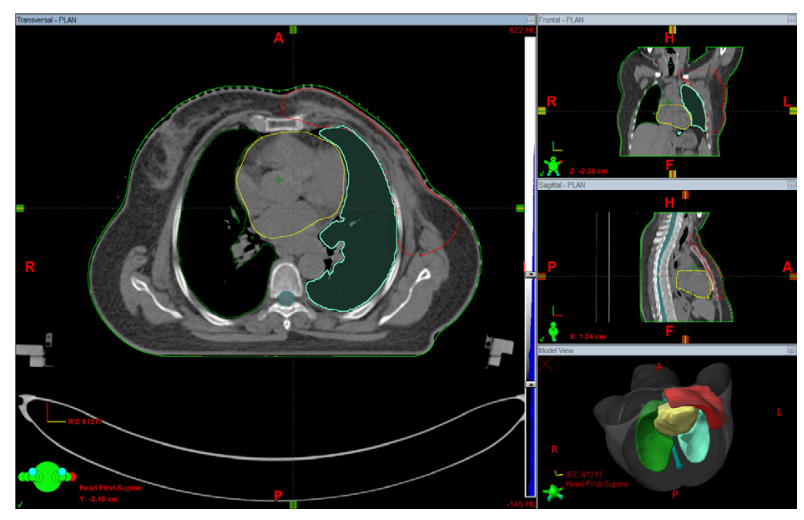

Figure 1. CT Image with contouring of PTV and OARs
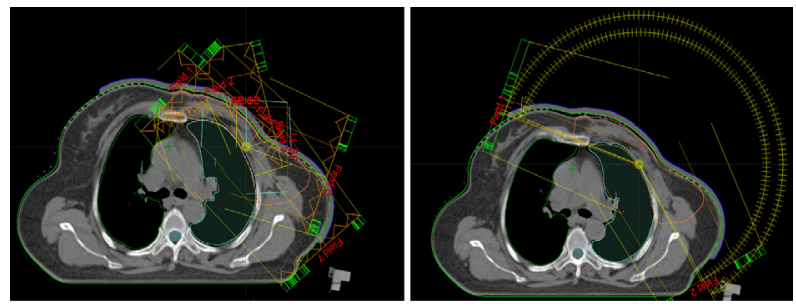

Figure 2. Beam Arrangement of IMRT plan (Left) and Arc arrangement for VMAT Plan (Right)

Table 1. Average Dose Histogram Parameters for both VMAT and IMRT Plans for all 20 Patients

\begin{tabular}{|c|c|c|c|c|}
\hline \multirow[b]{2}{*}{ Organ } & \multicolumn{2}{|c|}{ V MAT } & \multicolumn{2}{|c|}{ IMRT } \\
\hline & Mean & SD & Mean & SD \\
\hline \multicolumn{5}{|l|}{ PTV } \\
\hline CI & 1.05 & 0.02 & 1.07 & 0.03 \\
\hline HI & 0.065 & 0.005 & 0.069 & 0.008 \\
\hline \multicolumn{5}{|l|}{ Heart } \\
\hline D - $20 \%$ V (cGy) & 960 & 205 & 1300 & 261 \\
\hline Mean Dose (cGy) & 1236 & 232 & 1870 & 298 \\
\hline \multicolumn{5}{|l|}{ Left Lung } \\
\hline Mean Dose (cGy) & 1205 & 301 & 1435 & 359 \\
\hline D - 5\%V (cGy) & 3395 & 396 & 3525 & 565 \\
\hline D - $20 \% \mathrm{~V}$ (cGy) & 1840 & 315 & 2012 & 406 \\
\hline \multicolumn{5}{|l|}{ Right Lung } \\
\hline Mean Dose (cGy) & 505 & 138 & 553 & 165 \\
\hline MU (MU) & 512 & 59.5 & 1170 & 215 \\
\hline NTID $\left(\times 10^{5} \mathrm{Gycm}^{3}\right)$ & 108.8 & 20.5 & 110.1 & 24.1 \\
\hline
\end{tabular}

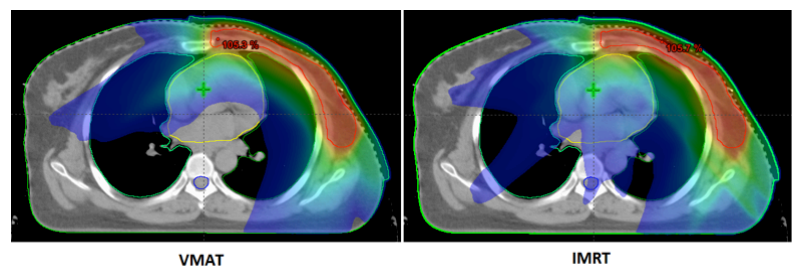

Figure 3. Iso Dose Distribution of both VMAT and IMRT Plans

DMX (Varian Medical Systems, Palo Alto, California, USA) integrated with 120 leaves millennium multi leaf collimator was used. Both Intensity modulated Radiotherapy Plans as well as VMAT plans were generated for these patients.

IMRT Planning: IMRT plans with 7 beams in an arc 


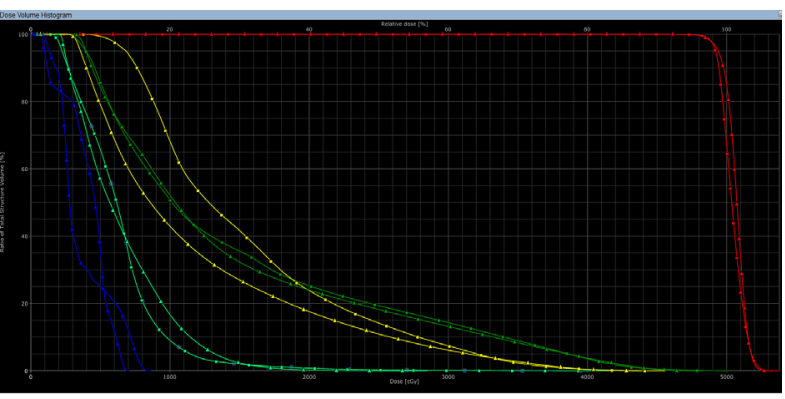

Figure 4. DVH Comparison of both VMAT and IMRT Plan for one Patient Selected Randomly among All 20 Patients

fashion were used to achieve the criteria of $95 \%$ of the prescribed dose to the PTV. In Eclipse TPS, the IMRT plans were created with inverse plan optimization with Dose volume optimizer and Analytical Anisotropic Algorithm (AAA) for dose calculation. Leaf motion calculator was used to calculate the leaf motions.

VMAT Planning: VMAT plans with two semi arcs were used to achieve $95 \%$ coverage to the PTV. The VMAT plans were created with inverse plan optimization with Progressive resolution optimizer (PRO) and Analytical Anisotropic Algorithm (AAA) for dose calculation.

Both IMRT and VMAT plans were made for a total dose of $5000 \mathrm{cGy}$ in which $4000 \mathrm{cGy}$ was delivered with bolus and 1000cGy was delivered without bolus to reduce skin morbidity. For both IMRT as well as VMAT plans dose constraints were given so as to obtain an optimal plan.

The plans were evaluated using Dose volume Histograms. The target dose uniformity and conformity were calculated and evaluated.

\section{Results and Discussion}

The dose coverage, Homogeneity index, Conformity index, Ipsilateral Lung Dose, Heart dose, Contralateral Lung Dose, Monitor Units, Normal Tissue Integral Dose for all the 20 patients are averaged and the Standard deviation was also calculated. The same is tabulated below.

The tabular clearly depicts the mean and the standard deviation of the dose coverage obtained for the target volume and other critical structures for all the 20 patients. Also the mean Monitor units and Normal tissue Integral dose are tabulated above. The below discussion gives a clear description of the Table 1, target and critical structure wise (Mirza Athar et al., 2014)

\section{Target coverage}

The dose coverage obtained in VMAT is more uniform and homogeneous than that obtained with IMRT. The mean of the Conformity index in VMAT plans is found to be 1.05 while it is 1.07 in IMRT. The mean homogeneity index in VMAT plans was found to be 0.065 and it was 0.069 in IMRT plans.

\section{Heart dose}

The critical structures dose were also obtained from the Dose volume histograms and compared for both VMAT and IMRT plans. The mean of the dose to $20 \%$ volume of Heart was found to be 960 cGy in VMAT plans while it was 1300 cGy in IMRT plans. The mean dose to heart was $1236 \mathrm{cGy}$ in VMAT plans and it was $1870 \mathrm{cGy}$ in IMRT plans.

\section{Ipsilateral (Left) Lung Dose}

The dose to $5 \%$ volume and $20 \%$ volume, mean dose were observed and tabulated. In VMAT plans the mean 5\% volume dose was $3395 \mathrm{cGy}$ mean, $20 \%$ volume dose was $1840 \mathrm{cGy}$ and the mean of the mean dose was $1205 \mathrm{cGy}$. In IMRT plans, the mean $5 \%$ volume dose was $3525 \mathrm{cGy}$, $20 \%$ volume dose was $2012 \mathrm{cGy}$ and the mean dose was $1435 \mathrm{cGy}$.

\section{Contralateral (Right) Lung}

The mean dose was observed for the contralateral Lung. However the variation is less, the mean dose in VMAT plans was 505cGy and 553cGy in IMRT plans.

\section{Monitor Units}

The mean of the Monitor units for all the patients in VMAT plans was 512MU while it was 1170 in IMRT plans.

\section{Normal Tissue Integral Dose (NTID)}

The Normal Tissue integral dose is obtained using the mean dose to the body excluding the Planning Target volume and its volume. The NTID in VMAT plans is $108.8 \times 10^{5} \mathrm{Gycm}^{3}$ and $110.1 \times 10^{5} \mathrm{Gycm}^{3}$ in IMRT plans.

Conclusion: The target coverage, homogeneity and Conformity index are better in VMAT plans than the IMRT plans. The heart dose which is a major concern in the radiotherapy of especially in Left breast carcinoma received lesser dose in VMAT plans than in the IMRT plans. The Ipsilateral Lung dose was also very less in VMAT plans than the IMRT plans. The Contralateral Lung dose and the Normal Tissue Integral Dose were also lesser in VMAT plans however the difference is not very appreciable. The MU in VMAT plans is quite lesser than the IMRT plans which is a very great advantage because of the reduction in treatment time. On the whole VMAT seems to be a better modality of treatment in the radiotherapy of Left Breast carcinoma.

\section{Acknowledgements}

We acknowledge Dr.Rajesh Jeganathan, Managing Director, Billroth Hospitals Ltd, Chennai, India, for providing the facility to carry out this work. We also acknowledge Dr.Saritha Damodaran, Radiation Oncologist and Ms.R.Tharani, Medical Physicist, Billroth Hospitals Ltd Chennai, India, for their support.

\section{References}

Ali MA, Babaiah M, Madhusudhan N, et al (2014). Comparative dosimetric analysis of IMRT and VMAT (RapidArc) in brain, head and neck, breast and prostate malignancies. Int J Cancer Ther Oncol, 3, 3019.

Ekambaram V, Shiyama S, Velayudham R, et al (2014). Biological 
evaluation of 3 dimensional conformal radiotherapy and intensity modulated radiotherapy plans in different clinical situations. JIPMER J Cancer, 3, 35-43.

Ekambaram V, Velayudham R (2013) Commissioning and Acceptance Testing of the existing linear accelerator upgraded to volumetric modulated arc therapy; Rep Pract Oncol Radiother, 18, 286-97.

Ekambaram V, Velayudham R (2014). Analysis of low dose level volumes in intensity modulated radiotherapy and 3-D conformal radiotherapy. Int J Cancer Ther Oncol, 2, 2032.

Mansouri S, Naim A, Glaria L, et al (2014). Dosimetric evaluation of 3-D conformal and intensity-modulated radiotherapy for breast cancer after conservative surgery. Asian Pac J Cancer Prev, 15, 4727-32.

Moorthy S, Sakr H, Hasan S, et al (2013). Dosimetric study of SIB-IMRT versus SIB-3DCRT for breast cancer with breathhold gated technique. Int J Cancer Ther Oncol, 3, 10110.

Naqvi SA, Mohiuddin MM, Yu CX (2010). Comparing Radiation Treatments Using Intensity-Modulated Beams, Multiple Arcs and Single Arc. Int J Radiat Oncol Biol Phys, 76.

Nicolini G, Clivio A, Fogliata A, et al (2009) Simultaneous integrated boost radiotherapy for bilateral breast: a treatment planning and dosimetric comparison for volumetric modulated arc and fixed field intensity modulated therapy. Radiation Oncol, 4, 27

Rana S (2013) Intensity modulated radiation therapy versus volumetric intensity modulated arc therapy. J Medical Radiation Sci, 60, 81-83

Swamy ST, Radha CA, Kathirvel M, et al (2014). Feasibility study of deep inspiration breath-hold based volumetric modulated arc therapy for locally advanced left sided breast cancer patients. Asian Pac J Cancer Prev, 15, 9033-38.

Zhu W-G, Zhou K, Yu C-H, et al (2012). Efficacy analysis of simplified intensity-modulated radiotherapy with high or conventional dose and concurrent chemotherapy for patients with neck and upper thoracic esophageal carcinoma. Asian Pac J Cancer Prev, 13, 803-07. 